\title{
HOOK EFFECT: A RARE PHENOMENON IN MOLAR PREGNANCY
}

\author{
Neelam Nalini ${ }^{1}$
}

\section{HOW TO CITE THIS ARTICLE:}

Neelam Nalini. "Hook Effect: A Rare Phenomenon in Molar Pregnancy". Journal of Evolution of Medical and Dental Sciences 2015; Vol. 4, Issue 60, July 27; Page: 10549-10552, DOI: 10.14260/jemds/2015/1520

ABSTRACT: High level of $\beta$-hCG is characteristics of Molar Pregnancy. Molar pregnancy with false negative urine hCG, which is a rarity, is called "HOOK EFFECT". Here I am going to report a case of 28 weeks size Molar pregnancy, which was misdiagnosed as large fibroid with multiple cystic degenerative area by both obstetricians and radiologist. This happened due to false negative urine $\beta$ hCG test. So to avoid this, clinicians should always keep in their mind, rare possibility of HOOK EFFECT due to very high level of serum $\beta$-hCG and in a case of high degree of suspicion, test should be repeated after dilution of sample.

KEYWORDS: $\beta$-hCG (Beta-Human Chorionic Gonadotropin) Urine Pregnancy Test, False negative, Hydatidiform mole.

INTRODUCTION: Molar pregnancy, commonly known as Hydatidiform mole is very common entity in our country, especially in Jharkhand. It is a benign condition with malignant potential. It comprises, a broad spectrum of benign and malignant tumors derived from trophoblast of human placenta in early pregnancy.

Characteristics of molar pregnancy is production of large amount of human chorionic gonadotropin by these rapidly proliferating trophoblastic tissue. So why high level of serum beta $\beta$ hCG and positive urine pregnancy test is a common finding with rarest exception (hook effect). Confirmatory test is ultrasonography. The false negative result for beta $\beta$-hCG is due to very high level of serum beta $\beta$-hCG secreted by molar tissue which causes oversaturation of beta $\beta$-hCG immune assay system. Patient of Molar Pregnancy usually presents with history of amenorrhea of variable period or sometimes pre diagnosed early pregnancy (by urine pregnancy test) with irregular bleeding per vaginum. Here we are going to report a case demonstrating this phenomenon.

CASE REPORT: Mrs. XYZ, 45/F reported in our outdoor with C/o- irregular, bleeding per vaginum for last 4 months, feeling of weakness and palpitation for last 11/2 months. M/H: Previous cycles were more or less regular 4-5/24-28 days. 0/H: P 4+1, all vaginal home delivery with uneventful antenatal, intranatal and postnatal period, last child was 4 years old. She denied any contraception.

O/E: Pallor ++++, Pulse: 132/min, regular, chest - Bilateral clear. CVS-Tachycardia, No added sound, P/A: Midline lump of about 28-30 weeks size, nontender, firm in consistency, lower margin could not be reached. Clinically it seemed to be a uterine lump. External ballottement was absent. FHS was absent. In pelvic examination CX was soft, long, posterior, external os closed, uterus was 28 weeks size with absent internal ballottement. For further confirmation and to rule out pregnancy related complications, as a bed side investigation urine pregnancy test was done, which was negative.

As patient was in poor general condition, she was admitted with provisional diagnosis of fibroid uterus.

INVESTIGATIONS: HB -5gm\%; PCV; MCH; MCHC; SGPT: 52 IU/L; Serum creatinine-0.9 mg\%; TC12,500/mm3; N-70\%, L-28\%, E-04\%, B-01\%, M-00\%; BT-1min, 20sec: CT-3min 10 sec, Chest X-ray PA 


\section{CASE REPORT}

view-Increased Broncho vascular markings. USG of whole abdomen: enlarged uterus with heterogeneous echogenicity lesion along endometrial cavity with multiple cystic/necrotic areas. Color spectral Doppler shows a vascular lesion, likely degenerated fibroid (Figure 1).

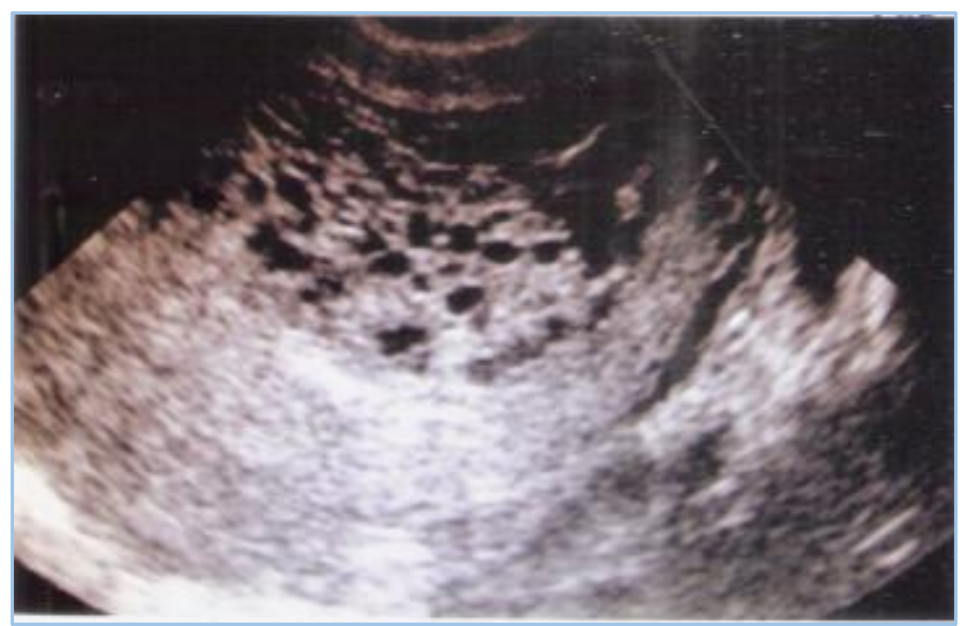

Figure 1: USG film showing large heterogeneous mass with multiple cystic/nectrotic areas

Patient was counseled and planned for hysterectomy after improvement of the general condition of the patient. Two units of blood were given. Suddenly after 4 days of admission, at night she was shifted to emergency with profuse bleeding per vaginum. At that time, Pallor - ++++, Pulse: 140/min, B. P. - 80/60 mmHg, Uterus was 16 weeks size. P/V: OS was open with bleeding +++ and grapes like molar tissue were found in vagina.

As it was pathognomonic of molar pregnancy. So patient was resuscitated and with 2 units of blood, shifted to emergency OT and suction and evacuation was done and profuse amount of molar tissue evacuated (Figure 2).

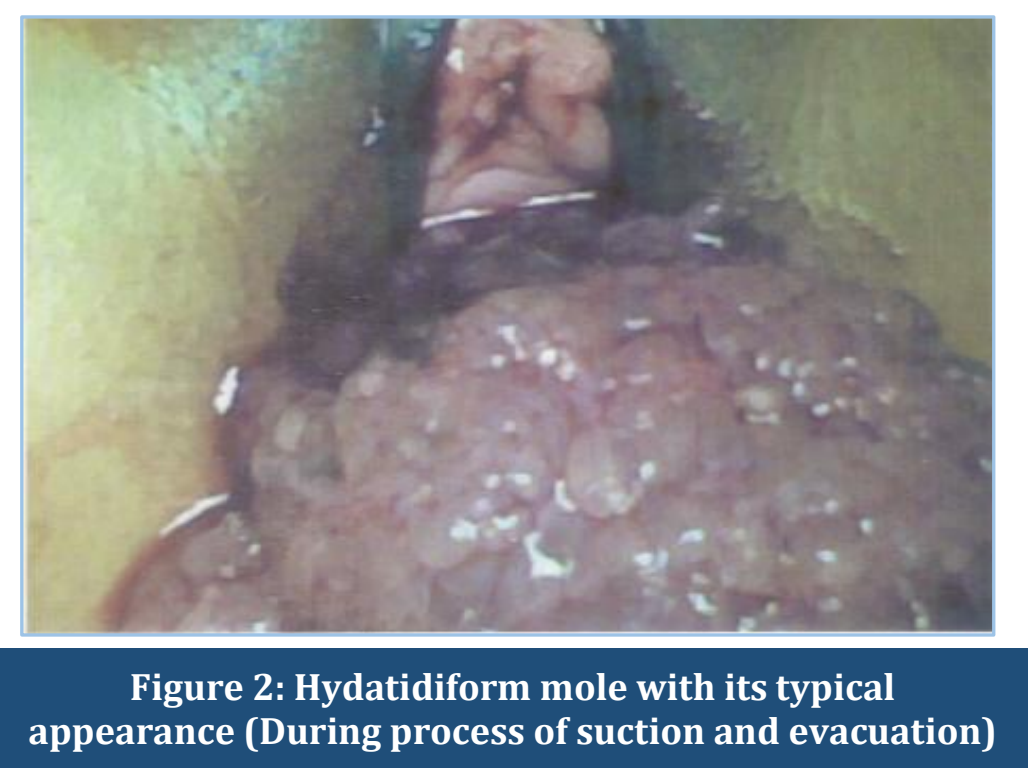


At the end of procedure uterus was 8 weeks size, contracted with slight bleeding P/V. Further 2 units of blood was given. Patient was kept under "molar pregnancy follow - up schedule". On next morning, blood sample for serum beta $\beta$-hCG was sent retrospectively with a request tag to do the test after dilution. The report was serum beta $\beta$-hCG 40,00,000IU/L. The patient was discharged after four days on repeated request and a strict guideline for follow up was provided.

But she was not well complaint and returned back only after four weeks for follow up with complain of bleeding per vaginum, more or less continuous since evacuation. At this time serum $\beta$-hCG was 20,000 IU/L. In USG uterus was $14 \mathrm{Cm}$ x $8 \mathrm{Cm}$ x $8.2 \mathrm{Cm}$ with retained product of conception. After detail counseling total abdominal hysterectomy plus bilateral salpingo-oophorectomy was done in a planned way. Post operatively, after forty days $\beta$-hCG disappeared from serum.

DISCUSSION: There is wide range of geographic and ethnic variation of the prevalence of molar pregnancy. The highest incidence is in Philippines being one in eighty pregnancies and lowest in European countries one in seven hundred fifty two. The incidence in India, is about one in four hundred.

In our case, patient was in reproductive age group with c/o bleeding P/V. Although in clinical examination, uterus was 28 week size with no foetal parts palpable, absent external and internal ballotment, and absent FHS; we still performed urine pregnancy test which was negative. So we excluded pregnancy related complication and wrongly jumped to the provisional diagnosis of fibroid uterus. Our diagnosis was further wrongly strengthened by diagnosis of degenerated fibroid in USG.

Probably, fluid filled vesicles, were misinterpreted by radiologists as multiple cystic degenerative area in a very large sized fibroid. We missed the diagnosis, because we didn't perform the test with diluted urine sample.

When I discussed this case retrospectively with concerned radiologist, he told that he had in his mind two differential diagnosis, molar pregnancy and large fibroid with multiple cystic degenerative area. But when he was told by post graduate doctor that urine pregnancy test for $\beta$-hCG was negative, he had also given the diagnosis of large fibroid with multiple degenerative area.

Here the purpose of this case report is to highlight "that clinicians should always keep in the corner of their mind the possibility (However rare) of "HOOK EFFECT" or "PROZONE PHENOMENON". Clinicians should rely on clinical acumen in such tricky situations.

Current urine and serum pregnancy tests use antibodies directed against $\beta$-hCG for immunologic identification. Two antibodies directed against different parts of the beta chain are used, and the resultant "sandwich" they form around the antigen is detected and interpreted as "positive". Most test kits have one antibody to site beta one and another to the C-terminus of the beta-subunit ( $\beta$ CTP), or to the alpha-subunit. However, falsely low or negative results can occur when an extremely high level of $\beta$-subunit core fragment, a breakdown product of $\beta$-hCG, overwhelms the assay system and blocks the formation of "sandwiches" between the two sites. ${ }^{1,2}$

This is known as the "hook effect" or "prozone phenomenon", and can be observed in immunometric sandwich assays.

However, it is important to note that hook effects occurring in both qualitative urine and quantitative serum $\beta$-hCG assays have been reported. ${ }^{3}$ Therefore, it is possible that if the actual serum beta- $\beta$-hCG is extremely high, the reported serum $\beta$-hCG may be spuriously underestimated due to the hook effect. 
This hook effect has also been documented with immunoassays with ferritin, prostate-specific antigen, prolactin, rapid plasma reagin for syphilis. ${ }^{4}$ thyrotropin and CA-125. It can be overcome by dilution of the test sample if suspicion is high.

Another learning point is the need to advocate close follow-up for post-molar GTT, especially in older women. In women over 35 years of age, the risk of post-molar GTT after suction evacuation appears to have increased and has been reported to be as high as $56 \%$ in women over 50 years of age ${ }^{5}$.

CONCLUSION: Although today we are equipped with high resolution ultrasound machine and modern immunological assay method with much improved reliability, clinicians should still be aware of the potential for false negative urinary and serum $\beta$-hCG results due to the high dose "HOOK EFFECT", especially in the setting of hydatidiform mole and delayed pregnancy test. Negative or inconclusive results in patient with high suspicious of pregnancy should be further evaluated by serum quantification of $\beta$-hCG after appropriate sample dilution.

\section{REFERENCES:}

1. Er-TK, Tsai LY, Gines Ruiz MA, et al Quantitative human chorionic gonadotropin measurement in urine using the Access immunoassay. Am J Emerg Med 2008; 26:103-4.

2. Yunus D, Muppala H, Hamer F, Clarke F. Three consecutive false negative pregnancy tests in a twin pregnancy, a case report. Int J Gynecol Obstet [serial online] 2007; 6. Available at: www. ispub.com/journal/the internet Journal_of_gynecology_and_obstetrics/volume_6_ number_2_6/article/three_consecutive_false_negative_pregnancy_test_a_twin_pregnancy_a_ case_report. Html. Accessed February 10, 2010.

3. Er TK, Jong YJ, Tsai EM, et al. False-negative pregnancy test in hydatidiform mole. Clin Chem 2006; 52:1616-8.

4. El-Zaatari MM, Martens MG, Anderson GD. Incidence of the prozone phenomenon in syphilis serology. Obstet Gynecol 1994; 84:609-12.

5. Tstikamoto N, Iwasaka T, Kashimura Y, et al. Gestational trophoblastic disease in women aged 50 or more. Gynecol Oncol 1985:20:53-61.

\section{AUTHORS:}

1. Neelam Nalini

\section{PARTICULARS OF CONTRIBUTORS:}

1. Assistant Professor. Department of Obstetrics and Gynaecology, Rajendra Institute of Medical Sciences, Ranchi.

FINANCIAL OR OTHER

COMPETING INTERESTS: None
NAME ADDRESS EMAIL ID OF THE CORRESPONDING AUTHOR:

Dr. Neelam Nalini, Address: E-159, Sector-2, Dhurwa, Ranchi-834004, Jharkhand, India.

Email: endmasingh@gmail.com

Date of Submission: 16/06/2015. Date of Peer Review: 17/06/2015. Date of Acceptance: 01/07/2015. Date of Publishing: 27/07/2015. 